\title{
La calidad de certificación de la mortalidad en Colombia, 2002-2006
}

Colombian death certificate quality, 2002-2006

\author{
Ricardo Cendales y Constanza Pardo \\ Instituto Nacional de Cancerología. Bogotá, Colombia. acardocen@yahoo.com, cpardo@cancer.gov.co \\ Recibido 17 Julio 2010/Enviado para Modificación 3 Enero 2011/Aceptado 4 Abril 2011
}

\section{RESUMEN}

Objetivo El estudio de la mortalidad resulta útil en la estimación de casos incidentes de cáncer; sin embargo se requiere evaluar la calidad de la certificación de la mortalidad antes de proceder a hacer las estimaciones de incidencia. El objetivo de este estudio es evaluar la calidad de la certificación de la mortalidad en Colombia.

Materiales y métodos Se analizó la calidad de la certificación de la mortalidad general y por cáncer en Colombia en el quinquenio 2002-2006. Se describieron indicadores clásicos de validez por medio de porcentajes simples para cada Departamento y además para cuatro áreas en donde operan registros poblacionales de cáncer (el área metropolitana de Bucaramanga y las ciudades de Cali, Manizales y Pasto). Se realizó un análisis de componentes principales con el fin de identificar relaciones no evidentes en el análisis descriptivo.

Resultados Se analizaron 952104 registros de defunción dentro de los cuales había 150163 muertes por cáncer. El 92,8\% de la mortalidad general estaba bien certificada. Predominaron los errores debidos al mal diligenciamiento. El 91,5\% de la mortalidad por cáncer estaba bien certificada. Predominaron los errores debidos a la mala definición de la localización del tumor primario como causa de defunción.

Conclusión La calidad de la certificación de la mortalidad general y por cáncer en Colombia es buena y ante la baja magnitud de problemas en la certificación, se puede emplear los datos de mortalidad en las estimaciones de incidencia de cáncer, sin aplicar más correcciones que las que se hacen de manera estándar.

Palabras Clave: Recolección de datos, estadísticas vitales, causas de muerte, países en desarrollo, control de calidad (fuente: DeCS, BIREME).

\section{ABSTRACT}

Objective Mortality study is useful for the estimation of cancer incident cases; but a quality assessment of the mortality information is required previous to the process of cancer incidence estimation. The aim of this study is to evaluate the quality of deathcertification in Colombia.

Materials and methods Overall quality of death certification and quality of cancer mortality certification was analyzed for the period 2002-2006. Classic validity indexes were 
described through simple percentages for each district and four cities, where operating population-based cancer registries: Bucaramanga, Cali, Manizales, and Pasto. A principal component analysis was performed in order to identify relationships that might not be evident in the descriptive analysis.

Results 952104 registries were analyzed, 150163 corresponding to cancer deaths. A high quality index was observed for overall death certification, with $92.8 \%$ of the overall mortality properly certified. Most errors were due to ill defined causes of death. A high quality index was also observed for cancer death registration with $91.5 \%$ of the cancer deaths properly certified. III-defined, secondary and unspecified cancer sites certification prevailed as the most frequent causes of error.

Conclusion Colombia has high quality standards for overall death and cancer death certification. Mortality data can be used confidently in the cancer incidence estimation process, without requirements for any specific corrections.

Key Words: Data collection, vital statistics, cause of death, developing countries, quality control (source: $\mathrm{MeSH}, \mathrm{NLM}$ ).

$\mathrm{E}$ estudio de la mortalidad resulta útil en el análisis de situación en salud, el seguimiento de los resultados de los programas de salud pública, la planeación de necesidades en servicios sanitarios y, en el caso particular del cáncer, en la estimación de incidencia. Se requiere evaluar la calidad de la certificación de la mortalidad antes de iniciar la estimación de incidencia.

La evaluación de la calidad de certificación de la mortalidad incluye varios componentes; aunque tradicionalmente se habían evaluado factores como la cobertura del sistema, la proporción de muertes certificadas por personal médico y la proporción de muertes por causas desconocidas o mal definidas $(1,2)$, también se ha evaluado la calidad de la certificación a través de la comparación de la causa básica de defunción que aparece en el certificado con la posible causa básica de defunción obtenida a partir del análisis de la historia clínica (3), de la información de la autopsia o de la autopsia verbal $(4,5)$.

Otros estudios han empleado un marco conceptual más amplio para la evaluación de la calidad de la certificación de la mortalidad (6); este incluye varios aspectos relacionados con la evaluación del potencial de generalización de los datos, la confiabilidad y validez de los datos y el uso de la información en la generación de políticas (7). La validez de los datos se evalúa a través de cinco aspectos: la proporción de muertes certificadas por no médicos, la proporción de muertes sin edad o sexo, la comparación de la causa registrada de mortalidad con la de un patrón de oro tal como la autopsia o la información de la historia clínica, la proporción de muertes registradas como de causas desconocidas o inespecíficas y la proporción de muertes con inconsistencias al respecto del sexo o la edad (8). 
La evaluación de la calidad de la certificación de la mortalidad por cáncer comparte varios de los aspectos ya descritos; dentro de estos se incluyen la evaluación de la validez a través de la comparación de la causa de mortalidad con la localización del sitio del tumor primario registrada en otras fuentes confiables $(9,10)$; aunque también incorpora algunos otros indicadores más específicos como el porcentaje de muertes por cáncer de sitios mal definidos (11) y el porcentaje de muertes por cáncer de útero de sitio no especificado (12).

Este estudio evalúa la calidad de la certificación de la mortalidad en Colombia, en el periodo 2002-2006, a través de indicadores clásicos de validez, haciendo énfasis en la calidad de la certificación de la mortalidad por cáncer. Los resultados son un insumo para la estimación de incidencia por cáncer en Colombia, pues permiten caracterizar la magnitud de los problemas en la certificación.

\section{MATERIALES Y MÉTODOS}

Se planteó un estudio descriptivo, con base en el análisis del universo de los certificados de defunción registrados en las bases de datos de mortalidad nacional. Los resultados se presentan para cada Departamento y además para el área metropolitana de Bucaramanga (conformada por los municipios de Bucaramanga, Girón, Floridablanca y Piedecuesta) y las ciudades de Cali, Manizales y Pasto. En estas áreas operan cuatro registros de cáncer de base poblacional que se están considerando como posible insumo para la estimación de casos incidentes de cáncer en Colombia. Todos los análisis se hicieron con base en el área de ocurrencia de la defunción.

Fuentes de información. Se obtuvieron las bases de datos oficiales de mortalidad del Departamento Administrativo Nacional de Estadística (DANE) para el quinquenio 2002-2006.

Variables consideradas en la evaluación general de calidad de la certificación de la mortalidad general. Se describieron las muertes que no fueron certificadas por médicos, las muertes que fueron registradas como signos, síntomas y afecciones mal definidas (R00-R99), las muertes sin información de edad, las lesiones de intencionalidad indeterminada (Y10-Y34,Y87.2), las muertes cardiovasculares de etiología mal definida (I47.2, I49.0, I46, I50, I51.4, I51.5, I51.6, I51.9, I70.9) y las muertes por cáncer certificadas como de primario no establecido, de sitios mal especificados o como consecuencia de tumores metastáticos (C76-C80,C97). 
Variables consideradas en la evaluación de calidad de la certificación de la mortalidad por cáncer. Se describieron las muertes por cáncer que no fueron certificadas por médico, las muertes por cáncer de primario no establecido, de sitios mal especificados o como consecuencia de un tumor metastático sin un primario establecido (C76-C80, C97); las muertes por cáncer sin información de edad y las muertes por cáncer de útero de sitio no especificado (C55).

Métodos estadísticos. Un único registro puede tener varios problemas relacionados con la calidad de la certificación; por ejemplo, puede tener una causa de muerte mal definida, puede no tener información de edad y además puede que no haya sido certificado por un médico. En esta situación, si se le da similar relevancia a cada uno de estos aspectos tendrían que describirse los tres problemas mencionados de manera independiente y un mismo registro aparecería descrito en varios indicadores a la vez. Sin embargo, en los resultados no se presenta la descripción independiente de los tres problemas, pues el análisis pretende describir la magnitud del problema de la certificación de forma jerárquica. Para que una causa de muerte sea válida en el contexto de los análisis de calidad de la certificación, primero debe ser certificada por un médico, luego debe tener la edad del fallecido y finalmente, debe tener una causa de defunción bien definida. Por lo tanto, una muerte con causa de defunción bien definida, con información de edad, pero que no ha sido certificada por un médico pierde validez. Por ello, para lograr hacer una descripción jerárquica del problema, primero se describieron las muertes que no habían sido certificadas por un médico; luego, entre las muertes que habían sido certificadas por médicos, se describieron las que no tenían información de edad, y finalmente, entre las muertes que habían sido certificadas por médicos y que tenían información de edad, se describieron las que tenían causas de muerte mal definidas.

La descripción de los indicadores de validez se hizo con el enfoque jerárquico descrito, a través de porcentajes simples. Se realizó un análisis factorial de componentes principales con el fin de detectar una posible estructura de relación subyacente, tanto para el análisis general de la calidad de certificación como para el análisis de la certificación de la mortalidad por cáncer; se empleó el estadístico de Kaiser-Meyer-Olkin y la prueba de esfericidad de Bartlett con el fin de identificar si existía una estructura de relación subyacente; aquellos factores que no se ajustaban con las solución propuesta de factores principales fueron excluidos del análisis; el número de factores se seleccionó con ayuda del análisis gráfico; en aquellos casos en los que se consideró apropiado se hizo una rotación ortogonal utilizando el análisis de componentes principales como 
método de extracción y el varimax con normalización de Kaiser como método de rotación a partir del análisis gráfico. Los datos se procesaron con el software SPSS versión 15.0.

\section{RESULTADOS}

Calidad de la certificación de la mortalidad general

Se analizaron 952104 registros de defunción correspondientes al quinquenio 2002-2006. El porcentaje de muertes debidamente certificadas superó el $90 \%$; las dos causas que más afectaron la calidad de la certificación general de la mortalidad fueron los signos, síntomas y otras afecciones mal definidas y las muertes cardiovasculares mal definidas. El análisis desagregado mostró 10 Departamentos con un porcentaje de muertes debidamente certificadas inferior al $89 \%$. Vaupés es el Departamento que mostró más bajos indicadores en la calidad general de la certificación. Para la calidad de la certificación general de la mortalidad en las áreas de influencia de los registros poblacionales de cáncer de Bucaramanga, Cali, Manizales y Pasto se analizaron 143515 defunciones lo cual correspondió al $15,1 \%$ de la mortalidad nacional. La calidad de la certificación de la mortalidad en estas cuatro áreas fue superior a las cifras observadas en Colombia (Tabla 1).

El análisis de componentes principales reveló a través del estadístico de Kaiser-Meyer-Olkin (valor KMO de 0,664) y de la prueba de esfericidad de Bartlett (valor $\mathrm{p}<0,0001$ ) que la varianza común podría estar explicada por factores subyacentes; el análisis de la varianza total explicada, junto con el análisis gráfico del scree plot, demostraron que dos componentes principales pueden explicar la falta de calidad en un $71 \%$ : el primero está relacionado con la falta de certificación por un médico y la certificación inadecuada de la muerte como un signo, síntoma o afección mal definida (Amazonas, Cauca, Nariño, Vaupés); el segundo tiene que ver con la certificación inadecuada de las muertes violentas y la falta de diligenciamiento de la edad (Caquetá, Casanare, Guaviare, Meta, Putumayo, Vichada). El primer componente también determina un grupo de Departamentos en el que a pesar de tener certificación por médico hay errores en la certificación de las muertes por cáncer (Antioquia, Bogotá, Caldas, Quindío, Risaralda); de manera similar, el segundo componente determina un grupo de Departamentos en los que a pesar de una buena certificación de la edad y de las muertes violentas, hay una inadecuada certificación de las muertes cardiovasculares (Arauca, Bolívar, Boyacá, Cundinamarca, Norte de Santander, Santander, Sucre y Tolima) (Tabla 1, Figura 1). 
Figura 1.Análisis de componentes principales en la evaluación general de la calidad de la certificación de la mortalidad (Figura rotada), Colombia, 2002-2006

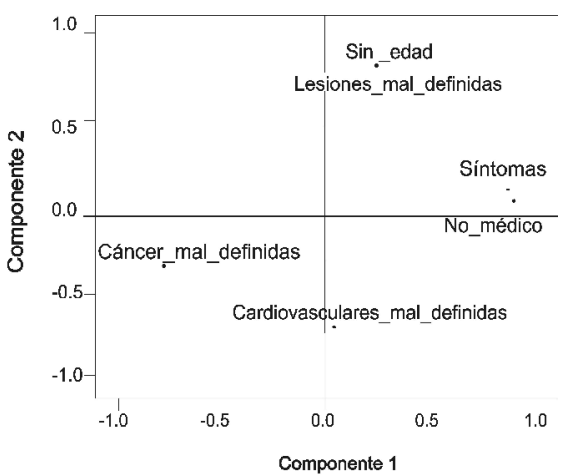

Tabla 1. Indicadores (\%) de calidad de la certificación general de la mortalidad según Departamento de ocurrencia de la defunción. Colombia, 2002-2006

\begin{tabular}{|c|c|c|c|c|c|c|c|}
\hline Departamento & $\begin{array}{c}\text { Bien } \\
\text { certificadas }\end{array}$ & $\begin{array}{c}\text { No } \\
\text { certificadas } \\
\text { por médico }\end{array}$ & $\begin{array}{l}\text { Sin } \\
\text { edad }\end{array}$ & $\begin{array}{c}\text { Causas } \\
\text { mal } \\
\text { definidas }\end{array}$ & $\begin{array}{l}\text { Lesiones de } \\
\text { intención no } \\
\text { determinada }\end{array}$ & $\begin{array}{c}\text { Cardio } \\
\text { vasculares mal } \\
\text { definidas }\end{array}$ & $\begin{array}{l}\text { Cáncer sin } \\
\text { primario } \\
\text { establecido }\end{array}$ \\
\hline Antioquia & 94,1 & 0,1 & 1,1 & 1,3 & 1,0 & 1,3 & 1,2 \\
\hline Atlántico & 94,7 & 0,0 & 1,2 & 0,9 & 0,2 & 2,3 & 0,8 \\
\hline Bogotá D.C. & 92,7 & 0,0 & 0,0 & 2,7 & 1,7 & 1,6 & 1,3 \\
\hline Bolívar & 93,6 & 0,7 & 0,4 & 1,4 & 0,2 & 2,6 & 1,0 \\
\hline Boyacá & 92,7 & 0,4 & 0,2 & 1,3 & 1,2 & 3,3 & 0,9 \\
\hline Caldas & 94,9 & 0,0 & 0,4 & 0,7 & 0,5 & 2,2 & 1,2 \\
\hline - Manizales & 95,8 & 0,0 & 0,4 & 0,5 & 0,3 & 1,9 & 1,2 \\
\hline Caquetá & 88,0 & 0,7 & 2,8 & 2,0 & 4,0 & 1,9 & 0,6 \\
\hline Cauca & 83,5 & 9,3 & 0,9 & 2,3 & 1,6 & 1,7 & 0,8 \\
\hline Cesar & 93,5 & 0,4 & 2,4 & 1,0 & 0,2 & 1,8 & 0,7 \\
\hline Córdoba & 92,7 & 1,3 & 1,0 & 1,2 & 0,6 & 2,2 & 0,9 \\
\hline Cundinamarca & 92,2 & 0,0 & 1,0 & 1,8 & 1,4 & 2,9 & 0,8 \\
\hline Chocó & 89,3 & 3,7 & 1,4 & 2,2 & 1,2 & 1,6 & 0,7 \\
\hline Huila & 93,2 & 0,2 & 0,4 & 1,6 & 1,5 & 2,3 & 0,9 \\
\hline La Guajira & 92,0 & 2,9 & 1,7 & 1,1 & 0,2 & 1,4 & 0,5 \\
\hline Magdalena & 92,1 & 1,5 & 1,6 & 1,5 & 0,2 & 2,3 & 0,8 \\
\hline Meta & 91,7 & 0,0 & 2,1 & 1,1 & 2,1 & 2,1 & 0,9 \\
\hline Nariño & 87,2 & 5,1 & 0,9 & 1,9 & 2,2 & 2,2 & 0,6 \\
\hline - Pasto & 94,3 & 0,4 & 0,5 & 0,6 & 1,7 & 1,8 & 0,6 \\
\hline Norte de Santander & 89,0 & 0,4 & 0,9 & 2,2 & 0,8 & 5,6 & 1,0 \\
\hline Quindío & 96,2 & 0,0 & 0,1 & 0,2 & 0,1 & 2,0 & 1,4 \\
\hline Risaralda & 95,1 & 0,1 & 0,2 & 1,0 & 0,4 & 1,8 & 1,4 \\
\hline Santander & 90,9 & 0,8 & 0,9 & 1,8 & 0,8 & 3,7 & 0,9 \\
\hline - Bucaramanga ${ }^{a}$ & 93,8 & 0,2 & 0,5 & 1,2 & 0,5 & 2,7 & 1,1 \\
\hline Sucre & 90,3 & 1,7 & 1,9 & 1,4 & 0,3 & 3,4 & 0,9 \\
\hline Tolima & 94,0 & 0,8 & 0,5 & 0,8 & 0,5 & 2,4 & 0,9 \\
\hline Valle del Cauca & 95,4 & 0,2 & 0,4 & 0,6 & 0,6 & 1,7 & 1,0 \\
\hline - Cali & 96,0 & 0,0 & 0,5 & 0,4 & 0,6 & 1,6 & 1,1 \\
\hline Arauca & 93,5 & 0,1 & 0,9 & 0,8 & 1,4 & 2,6 & 0,6 \\
\hline Casanare & 87,9 & 0,1 & 3,1 & 1,4 & 4,2 & 2,4 & 0,8 \\
\hline Putumayo & 81,1 & 3,3 & 7,1 & 1,9 & 4,8 & 1,0 & 0,8 \\
\hline San Andrés y Prov. & 95,6 & 0,0 & 0,9 & 0,7 & 0,1 & 1,9 & 0,9 \\
\hline Amazonas & 88,6 & 2,4 & 1,9 & 2,0 & 2,8 & 1,7 & 0,6 \\
\hline Guainía & 81,7 & 10,7 & 1,6 & 0,5 & 2,5 & 2,5 & 0,5 \\
\hline Guaviare & 86,9 & 0,2 & 3,0 & 2,3 & 6,0 & 1,1 & 0,5 \\
\hline Vaupés & 66,9 & 19,6 & 2,5 & 6,1 & 3,1 & 1,8 & 0,0 \\
\hline Vichada & 88,3 & 3,2 & 1,8 & 1,6 & 3,4 & 1,4 & 0,5 \\
\hline Colombia & 92,8 & 0,7 & 0,8 & 1,5 & 1,1 & 2,1 & 1,0 \\
\hline
\end{tabular}


Calidad de la certificación de la mortalidad por cáncer

En la evaluación de la calidad de la certificación de la mortalidad por cáncer para Colombia, se consideraron 150163 muertes, de las cuales un 91,5\% estaban debidamente certificadas. Las muertes por cáncer de sitio mal definido y por cáncer de útero de sitio mal definido son las causas que más afectan la calidad de la certificación por cáncer en Colombia. El análisis desagregado mostró que 10 Departamentos tuvieron índices de calidad de certificación de mortalidad por cáncer inferior al $89 \%$. Cauca fue el Departamento con el índice más bajo de calidad. En las áreas de influencia de los registros poblacionales de cáncer de Bucaramanga, Cali, Manizales y Pasto (que sumaron 24405 defunciones por cáncer), la calidad de la certificación de la mortalidad por cáncer fue similar a las cifras observadas en Colombia (Tabla 2).

De igual forma, con el estadístico de Kaiser-Meyer-Olkin (valor KMO de 0,476), la prueba de esfericidad de Bartlett (valor p de 0,005 ) y el análisis gráfico de componentes principales, se concluyó que el $74 \%$ de la varianza común podría estar explicada por dos factores subyacentes: el primero está relacionado con la certificación de muertes por cáncer de sitio mal definido (Antioquia, Boyacá, Caldas, Norte de Santander, Quindío y Risaralda); el segundo tiene que ver con la falta de información de la edad y la certificación de muertes por cáncer de útero de sitio no especificado (Amazonas, Casanare, Córdoba, Magdalena, Putumayo, San Andrés y Providencia); el segundo componente también determina las muertes por cáncer que no son certificadas por un médico (Cauca, Chocó, Guajira, Nariño, Sucre, Vaupés, Vichada) (Tabla 2, Figura 2).

Figura 2. Análisis de componentes principales en la evaluación de la calidad de la certificación de la mortalidad por cáncer (Figura rotada), Colombia, 2002-2006

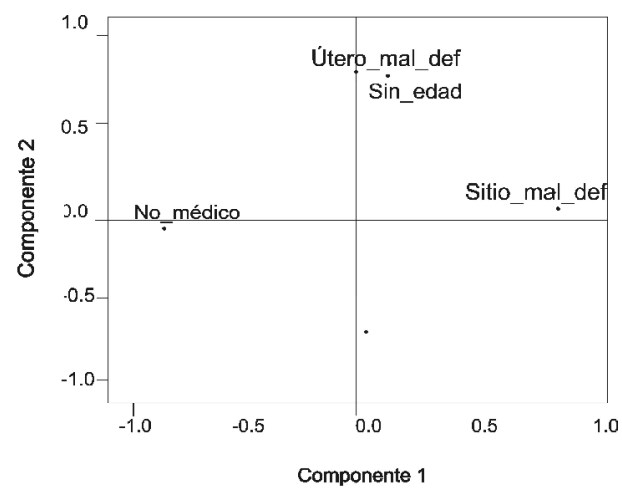


Tabla 2. Indicadores (\%) de calidad de la certificación de la mortalidad por cáncer según Departamento de ocurrencia de la defunción. Colombia, 2002-2006

\begin{tabular}{|c|c|c|c|c|c|}
\hline $\begin{array}{l}\text { Departamento de } \\
\text { ocurrencia }\end{array}$ & $\begin{array}{l}\text { Muertes por } \\
\text { cáncer bien } \\
\text { certificadas }\end{array}$ & $\begin{array}{c}\text { Muertes por } \\
\text { cáncer no } \\
\text { certificadas } \\
\text { por médico }\end{array}$ & $\begin{array}{l}\text { Muertes por } \\
\text { cáncer sin } \\
\text { información de } \\
\text { edad }\end{array}$ & $\begin{array}{l}\text { Muertes por } \\
\text { cáncer de } \\
\text { sitio mal } \\
\text { definido }\end{array}$ & $\begin{array}{l}\text { Muertes por } \\
\text { cáncer de útero } \\
\text { de sitio no } \\
\text { especificado }\end{array}$ \\
\hline Antioquia & 91,9 & 0,0 & 0,3 & 7,1 & 0,6 \\
\hline Atlántico & 92,4 & 0,0 & 1,1 & 5,0 & 1,6 \\
\hline Bogotá D.C. & 92,8 & 0,0 & 0,0 & 6,5 & 0,7 \\
\hline Bolívar & 90,6 & 0,8 & 0,1 & 6,7 & 1,7 \\
\hline Boyacá & 91,5 & 0,3 & 0,1 & 7,0 & 1,2 \\
\hline Caldas & 91,5 & 0,0 & 0,2 & 7,5 & 0,8 \\
\hline Manizales & 92,2 & 0,0 & 0,2 & 7,0 & 0,6 \\
\hline Caquetá & 90,2 & 0,8 & 1,1 & 6,3 & 1,6 \\
\hline Cauca & 83,2 & 9,6 & 0,5 & 5,3 & 1,3 \\
\hline Cesar & 90,4 & 0,7 & 0,6 & 5,7 & 2,5 \\
\hline Córdoba & 88,1 & 2,1 & 1,2 & 6,5 & 2,1 \\
\hline Cundinamarca & 91,4 & 0,0 & 0,8 & 6,5 & 1,3 \\
\hline Chocó & 84,6 & 5,4 & 0,6 & 7,8 & 1,5 \\
\hline Huila & 91,7 & 0,2 & 0,4 & 6,4 & 1,3 \\
\hline La Guajira & 86,0 & 4,2 & 1,7 & 5,5 & 2,6 \\
\hline Magdalena & 89,3 & 0,9 & 1,2 & 5,6 & 2,9 \\
\hline Meta & 91,4 & 0,1 & 0,5 & 6,8 & 1,2 \\
\hline Nariño & 89,9 & 4,0 & 0,7 & 4,1 & 1,3 \\
\hline Pasto & 94,3 & 0,5 & 0,5 & 3,8 & 0,9 \\
\hline $\begin{array}{l}\text { Norte de } \\
\text { Santander }\end{array}$ & 91,0 & 0,5 & 0,4 & 7,1 & 0,9 \\
\hline Quindío & 91,2 & 0,0 & 0,0 & 8,1 & 0,7 \\
\hline Risaralda & 90,9 & 0,0 & 0,0 & 8,1 & 1,0 \\
\hline Santander & 92,3 & 0,6 & 0,7 & 5,7 & 0,7 \\
\hline Bucaramanga $^{a}$ & 92,7 & 0,1 & 0,5 & 6,0 & 0,7 \\
\hline Sucre & 87,8 & 2,7 & 1,7 & 6,1 & 1,7 \\
\hline Tolima & 91,3 & 0,3 & 0,1 & 6,7 & 1,6 \\
\hline Valle del Cauca & 92,3 & 0,1 & 0,2 & 6,5 & 0,9 \\
\hline Cali & 92,7 & 0,0 & 0,2 & 6,5 & 0,5 \\
\hline Arauca & 91,1 & 0,0 & 0,0 & 6,9 & 2,0 \\
\hline Casanare & 89,0 & 0,0 & 2,1 & 7,2 & 1,7 \\
\hline Putumayo & 84,6 & 0,9 & 2,2 & 10,3 & 1,9 \\
\hline $\begin{array}{l}\text { San Andrés y } \\
\text { Providencia }\end{array}$ & 92,1 & 0,0 & 1,2 & 4,9 & 1,8 \\
\hline Amazonas & 86,3 & 0,0 & 1,4 & 5,5 & 6,8 \\
\hline Guainía & 93,1 & 0,0 & 0,0 & 6,9 & 0,0 \\
\hline Guaviare & 85,9 & 0,0 & 1,6 & 10,9 & 1,6 \\
\hline Vaupés & 88,9 & 11,1 & 0,0 & 0,0 & 0,0 \\
\hline Vichada & 86,2 & 3,4 & 0,0 & 6,9 & 3,4 \\
\hline Colombia & 91,5 & 0,6 & 0,4 & 6,5 & 1,1 \\
\hline
\end{tabular}

á́rea metropolitana de Bucaramanga (Bucaramanga, Floridablanca, Girón y Piedecuesta)

\section{DISCUSIÓN}

La calidad de la certificación de la mortalidad debe ser abordada de manera integral, considerando los preceptos teóricos descritos relacionados con la evaluación del potencial de generalización de los datos, la confiabilidad y validez de los datos, y el uso de la información en la generación de políticas (8). Este estudio describe la calidad de la certificación de la mortalidad de manera parcial, a través de la evaluación de la validez, y clasifica a Colombia como un país que genera datos de alta calidad 
(dentro de la perspectiva pura de la validez) de acuerdo con los puntos de corte establecidos por la Organización Mundial de la Salud (13) y confirma la calidad de certificación media alta de Colombia, en donde se emplea una clasificación internacional de enfermedades actualizada, con detalle de hasta 4 dígitos y un porcentaje de exhaustividad superior al $70 \%$ (14).

Colombia es un país con grandes contrastes culturales, sociales y económicos; esta diversidad se ve reflejada en la calidad de la certificación de la mortalidad. En la actualidad la falta de calidad secundaria a certificación por no médicos es baja y predominan los errores en la certificación debidos al mal diligenciamiento. Las estrategias orientadas a mejorar la calidad de la certificación deben ajustarse a los problemas identificados en cada región. Aunque el porcentaje de muertes mal certificadas es bajo, el impacto de la falta de calidad puede afectar la validez de los análisis de mortalidad en algunos Departamentos, en particular en lo que se refiere a las muertes por cáncer de sitio no especificado y por cáncer de útero de sitio no especificado.

En las regiones en las que hay un registro poblacional de cáncer disponible, los indicadores generales de la mortalidad resultaron ser sustancialmente mejores que en el resto del Departamento, por lo que se considera que la información de mortalidad en estas áreas es válida y sirve como insumo para la estimación de la razón incidencia mortalidad necesaria para la estimación de casos incidentes de cáncer, sin necesidad de hacer correcciones. El hecho de que la calidad de certificación por cáncer en las áreas de los registros sea similar a las cifras nacionales o a las cifras de los Departamentos de los cuales estas ciudades son sus respectivas capitales, demuestra que no se han hecho esfuerzos adicionales para mejorar la calidad de la certificación de la mortalidad en las áreas de influencia de los registros; esto debe cambiar pues en estas áreas la calidad de la información de la mortalidad por cáncer debería ser superior.

Estudios futuros de la validez de la calidad de certificación por cáncer en Colombia deben incluir la comparación de la causa de mortalidad con la localización del sitio primario consignada en otras fuentes, particularmente en las regiones en las que existe un registro poblacional de cáncer pues esta información se convierte en un insumo importante para el ajuste de la mortalidad $(3,11)$

Agradecimientos: Este estudio fue financiado por el gobierno de Colombia y el Ministerio de Protección Social a través de fondos de inversión del Instituto Nacional de Cancerología. 


\section{REFERENCIAS}

1. Núñez FML, Icaza NMG. Quality of Mortality statistics in Chile, 1997-2003. Rev Med Chil. 2006;134(9):1191-1196.

2. Paes NA. Quality of death statistics by unknown causes in Brazilian states. Rev Saude Publica. 2007;41(3):436-445.

3. Rao C, Yang G, Hu J, Ma J, Xia W, Lopez AD. Validation of cause-of-death statistics in urban China. Int J Epidemiol. 2007;36(3):642-651.

4. Yang G, Rao C, Ma J, Wang L, Wan X, Dubrovsky G, et al. Validation of verbal autopsy procedures for adult deaths in China. Int J Epidemiol. 2006;35(3):741-748.

5. Wang L, Yang G, Jiemin M, Rao C, Wan X, Dubrovsky G, et al. Evaluation of the quality of cause of death statistics in rural China using verbal autopsies. J Epidemiol Community Health. 2007;61(6):519-526.

6. Mahapatra P, Chalapati RPV. Cause of death reporting systems in India: a performance analysis. Natl Med J India. 2001;14(3):154-162.

7. França E, de Abreu DX, Rao C, Lopez AD. Evaluation of cause-of-death statistics for Brazil, 2002-2004. Int J Epidemiol. 2008;37(4):891-901.

8. Rao C, Lopez AD, Yang G, Begg S, Ma J. Evaluating national cause-of-death statistics: principles and application to the case of China. Bull World Health Organ. 2005;83(8):618625.

9. Sánchez GMV, Izquierdo FA, Beltrán FM, Bosch JFX, Viladiu QP. Trends in cervix cancer mortality in Catalonia, 1975-1992: analysis of death certificates and cancer registry of Girona. Gac Sanit. 1996;10(53):67-72.

10. Percy C, Stanek E, Gloeckler L. Accuracy of cancer death certificates and its effect on cancer mortality statistics. Am J Public Health. 1981;71(3):242-250.

11. Pérez-Gómez B, Aragonés N, Pollán M, Suárez B, Lope V, Llácer A, et al. Accuracy of cancer death certificates in Spain: a summary of available information. Gac Sanit. 2006;20 Suppl 3:42-51.

12. Cáffaro RM, Garau LII, Cabeza IE, Franch SP, Obrador AA. Validity of death certificates for cancer in Mallorca. Gac Sanit. 1995;9(48):166-173.

13. Mathers CD, Fat DM, Inoue M, Rao C, Lopez AD. Counting the dead and what they died from: an assessment of the global status of cause of death data. Bull World Health Organ. 2005;83(3):171-177.

14. Mahapatra P, Shibuya K, Lopez AD, Coullare F, Notzon FC, Rao C, et al; on behalf of the Monitoring Vital Events (MoVE) writing group. Civil registration systems and vital statistics: successes and missed opportunities. Lancet. 2007;370:1653-1663. 